\title{
Therapeutic Targets of Fingolimod (FTY720) in the Brains of Alzheimer's Disease Patients and the Associated Biological Mechanisms: a Network Pharmacology Study
}

\section{Pengqi Yin}

First Affiliated Hospital of Harbin Medical University https://orcid.org/0000-0002-3963-2094

\section{Yang Xue}

First Affiliated Hospital of Harbin Medical University

Guozhong Li ( $\nabla$ hmu_guozhongli@126.com)

Harbin Medical University

\section{Di Zhong}

First Affiliated Hospital of Harbin Medical University

Research

Keywords: Alzheimer's disease, Fingolimod, S1PR1, network pharmacology, GABA synapses

Posted Date: September 23rd, 2020

DOI: https://doi.org/10.21203/rs.3.rs-80664/v1

License: (c) (1) This work is licensed under a Creative Commons Attribution 4.0 International License.

Read Full License 


\section{Abstract}

\section{Background}

The sphingosine-1-phosphate receptor (S1PR) modulator fingolimod (FTY720), which is commonly used as an immunomodulator in multiple sclerosis treatment, was recently reported to be effective in the treatment of Alzheimer's disease (AD), but its mechanism of action remains elusive. In this study, network pharmacology methods were applied to detect the potential pharmacological mechanisms of fingolimod in the brains of these patients.

\section{Methods}

The pharmacological macromolecular targets of fingolimod and fingolimod phosphate were downloaded from Swisstarget. Systematic intersection analysis of the expression profiles of brain tissues (709 AD tissues and 534 control tissues) was performed, and AD-associated fingolimod targets (F-ADGs) in the frontal, temporal and entorhinal cortices were analysed. The Minimal Common Oncology Data Elements method was used to detect core F-ADGs. Gene function enrichment analyses of the core F-ADGs were performed. Correlation analyses were performed to identify core F-ADGs with expression levels that were associated with the severity of AD. CIBERSORTx (https://cibersortx.stanford.edu/) was used to estimate the percentages of various immune cell populations in the cortex of each AD patient.

Results

We found the fingolimod targets S1PR1, GNG3, GNG11 and GABBR2 were simultaneously detected in all three cortical regions. The core F-ADGs in all three cortical regions were enriched in similar biological processes, such as the $G$ protein-coupled receptor signalling pathway, synaptic transmission, and pathways related to the function of synapses, especially GABAergic synapses. Core F-ADGs in the temporal and entorhinal cortices were also enriched in functions related to calcium signalling and cholinergic synapses. Correlation analysis showed that the expression levels of 7 core F-ADGs (S1PR1, NPY, NPY2R, GNAI1, GNG5, FPR1 and GPR183) in the frontal cortex were correlated with the severity of $A D$. No differences in immune cell infiltration between $A D$ patients and normal controls were observed in any of the three cortical regions.

\section{Conclusions}

The pharmacological macromolecular targets of fingolimod and fingolimod phosphate, including S1PR1, were abnormally expressed in the cortices of multiple AD patients. The pharmacological effect of fingolimod on $A D$ may be attributed to its ability to regulate GABA synapses, calcium signalling, and cholinergic synapses, other than regulating immune cell infiltration.

\section{Introduction}


Fingolimod (FTY720), a sphingosine-1-phosphate receptor (S1PR) modulator, is the first oral drug approved by the Food and Drug Administration (FDA) for the treatment of relapsing-remitting multiple sclerosis (RRMS)[1]. Recently, fingolimod was reported to be effective in the treatment of Alzheimer's disease (AD)[2][3][4], but its mechanism of action remains elusive. The treatment effect of fingolimod in RRMS is commonly attributed to its ability to retain autoreactive lymphocytes in the lymph nodes and prevent them from entering the circulation and central nervous system (CNS) by downregulating the expression of S1PR1 on T lymphocytes[5][6]. However, fingolimod can also have a direct impact on the CNS. As fingolimod is a lipophilic molecule, it can cross the blood-brain barrier (BBB)[7][8] and has been proven to exert direct effects in the CNS, including inhibiting microglial activation, reducing astrocyte proliferation, reducing the loss of dendritic spines, and preventing excitotoxic neuronal death[9]. Since these pathophysiological processes have also been observed in $A D$, fingolimod is thought to have therapeutic effects on the pathological processes of AD. Moreover, since extensive inflammatory cell infiltration of the CNS occurs in multiple sclerosis but not in $A D[10]$, the therapeutic effect of fingolimod on $A D$ may be attributed more to a direct effect on the CNS than to the regulatory function of lymphocytes. Currently, whether the effect of fingolimod on brain tissues from AD patients is nonspecific or whether fingolimod specifically affects the pathogenic processes of $A D$ is unclear.

To date, most of the studies that have explored the mechanism of action of fingolimod in AD have focused on the effect of fingolimod on $\beta$-amyloid precursor protein (APP) metabolism and $\beta$-amyloid (A $\beta$ ) protein aggregation[11][12][13]. However, because sphingolipid and sphingolipid receptors, such as S1PRs, are widely distributed in the CNS[14], the effect of fingolimod on AD is likely multifactorial. Thus, it is necessary to study the function of fingolimod in $A D$ from more than one perspective. Furthermore, some studies have shown that fingolimod receptors and related downstream factors are differentially expressed in different cerebral regions due to differences in the activities of enzymes[15][16], indicating that studies on the effects of fingolimod in AD should be performed separately in each brain region. Network pharmacology methods provide new approaches for more comprehensively elucidating the mechanisms underlying the pharmacological effects of drugs in specific diseases[17]. In this study, we used network pharmacology methods to study the pharmacological mechanism underlying the treatment effect of fingolimod in AD patients.

\section{Materials And Methods}

\subsection{Study design and rationale}

In this study, we performed intersection analysis of the brain expression profiles of AD patients to identify AD-associated genes (ADGs) and compared these ADGs with fingolimod-associated genes (FGs) to obtain fingolimod-associated ADGs (F-ADGs). Then, biological analyses were performed, and correlations between the expression of F-ADGs and disease severity were evaluated. Immune cell infiltration was 
estimated by CIBERSORTx to explore the sources of the ADGs. A flow chart of the experimental method is shown in Figure 1.

\subsection{FGs}

The chemical structures of fingolimod and its metabolic product fingolimod phosphate were downloaded from PubChem (https://pubchem.ncbi.nlm.nih.gov/). SwissTargetPrediction [1][2]

(http://www.swisstargetprediction.ch/) was used to predict the pharmacological targets of fingolimod and fingolimod phosphate based on their chemical structures. Pharmacological targets with a feasibility $>0.1$ were identified. UniProt (https://www.UniProt.org/) was used to standardize the naming of the drug targets. STRING (version 11.0) was used to retrieve protein-protein interaction (PPI) information with the retrieval condition "Species" set to "Homo sapiens", the "confidence" set to "high (greater than 0.7)", and the maximum number of interactions for the " 1 st shell" and "2nd shell" set to "No more than 20 interactors". The target genes of fingolimod and fingolimod phosphate and the proteins that were predicted to interact with these target genes were collectively referred to as FGs.

\subsection{ADGs}

Original microarray data associated with $A D$ were retrieved from Gene Expression Omnibus (https://www.ncbi.nlm.nih.gov/geo/) by searching for the keyword "Alzheimer". Exhaustive and nonredundant data for the frontal cortex, temporal cortex and entorhinal cortex were retrieved. Data for the frontal cortex were retrieved from eight datasets (GSE12685, GSE36980, GSE48350, GSE5281, GSE53890, GSE66333, GSE33000, and GSE118553) from 266 AD patients and 423 normal controls. Data for the temporal cortex were retrieved from six datasets (GSE29652, GSE36980, GSE37263, GSE5281, GSE15222, and GSE118553) from 205 AD patients and 210 normal controls. Data for the entorhinal cortex were retrieved from five datasets (GSE26927, GSE26972, GSE48350, GSE5281, and GSE118553) from $63 \mathrm{AD}$ patients and 76 normal controls. All processed expression data from the same brain region were merged and then normalized with the "sva" package in R. Only genes (probes) for which less than $50 \%$ of the gene expression data was missing were retained. Then, the data from normal controls and AD patients were compared to identify ADGs. The "limma" package in R was used for differential expression analysis, and Benjamini-Hochberg's method was used to correct for multiple comparisons. A ffold changel $>1.2$ and $p<0.05$ were considered to indicate significantly differentially expressed genes.(Table 1 )

\subsection{F-ADGs and PPI analysis}

The FGs and ADGs in each cortical region were compared to identify F-ADGs. STRING (version 11.0) was used for PPI analysis. After repeats were eliminated, a "confidence score" $>0.7$ was set to design the PPI network.

\subsection{Analysis of the F-ADG PPI network to select molecular modules and core F-ADGs}

The Minimal Common Oncology Data Elements (MCODE) method was used for network analysis to select core molecular modules from the PPI network. The screening criterion for the core modules was 
twice the average degree of the "MCODE score". Degree cutoff was set to 2, node score cutoff set to 0.2 , $\mathrm{K}$-core was set to 2 and maximum depth was set to 100 .

\subsection{Gene function and pathway enrichment analysis of core F-ADGs.}

Gene Ontology (GO) analysis of biological processes was performed using DAVID (version 6.8)[3]. The thresholds were set as "Count 2, EASE 0.1", and "Display" was set as "Benjamini". Terms with p-values $<0.1$ were reported. A Kyoto Encyclopaedia of Genes and Genomes (KEGG) network was constructed in Cytoscape using ClueGO[4] to visualize the pathways associated with large modules of genes in a functionally grouped network. ClueGO is updatable based on the most recent KEGG data. Pathways with p-values $<0.1$ were reported.

\subsection{Correlation analysis of core F-ADG expression and the clinical severity of AD}

The frontal cortex expression profiles of 9 AD patients were correlated with their Mini-Mental State Exam (MMSE) scores, which ranged from 11 to 22. Pearson's correlation analysis was used to assess the correlation between the expression values of the core F-ADGs and the MMSE scores of the AD patients. $R>0.7$ indicated a very strong linear correlation, $0.5<R<=0.7$ indicated a significant linear correlation, $0.3<R<=0.5$ indicated a low linear correlation and $R<=0.3$ indicated no linear correlation.

\subsection{Analysis of immune cell infiltration in the cortices of $A D$ patients}

CIBERSORTx (https://cibersortx.stanford.edu/)[5] was used to estimate the percentages of various cell populations in the cortex of each $A D$ patient. A signature matrix of each cell type was generated with the default settings. Bulk RNA-seq data was deconvoluted using the signature matrix with S-batch correction to remove variances between different sequence platforms. Two-tailed unpaired Student's t-test was used to analyse differences in the abundances of different cell types between $A D$ and normal controls. The $p-$ values were correct for multiple testing using the Benjamini-Hochberg method. $\mathrm{P}<0.05$ was considered to indicate statistical significance.

\section{Results}

\section{FGs}

A total of 36 pharmacological fingolimod or fingolimod phosphate targets ( 4 confirmed and 32 predicted based on chemical structures) were identified from the TargetScan database. Using the STRING database, 890 proteins that interact with these targets were identified and designated FGs.

\section{ADGs}

A total of 2159 genes, which were differentially expressed in the frontal cortices of AD patients compared to those of healthy controls, were identified as ADGs; 1046 of these genes were upregulated and 1103 
were downregulated in AD patients compared to normal controls. A total of 1679 ADGs (937 upregulated genes and 742 downregulated genes) were identified in the temporal cortex, and 2940 ADGs (1630 upregulated genes and 1310 downregulated genes) were detected in the entorhinal cortex.

\section{F-ADGs}

Of the 2156 ADGs identified in the frontal cortex, 164 genes (100 upregulated genes and 64 downregulated genes) were FGs, suggesting that they are targets of fingolimod, and were designated FADGs. A total of 575 interactive edges were formed with 110 F-ADGs in the PPI network. A total of 118 FADGs (77 upregulated $A D G s$ and 41 downregulated $A D G s$ ) were identified in the temporal cortex, among which 113 genes formed 602 PPIs. In the entorhinal cortex, 173 F-ADGs (107 upregulated F-ADGs and 66 downregulated F-ADGs) were identified, and 157 genes formed 864 PPIs.

\section{Molecular modules of F-ADGs}

MCODE analysis was used to analyse the PPI network of the F-ADGs in each cortical region. The $110 \mathrm{~F}-$ ADGs identified in the frontal cortex were divided into 9 molecular modules, with Cluster 1, which contained 18 core F-ADGs, being defined as the core cluster. The 113 F-ADGs identified in the temporal cortex were categorized into 8 molecular modules, with Cluster 1 and Cluster 2, each of which consisted of 20 core F-ADGs, being defined as the core modules. The 157 F-ADGs in the entorhinal cortex were divided into 8 molecular modules, being Cluster 1 and Cluster 2, which each contained 32 core F-ADGs, being defined as the core modules. S1PR1, GNG3, GNG11 and GABBR2 were found in the core modules of all three cortical regions.

\section{Gene function enrichment analysis of core F-ADGs}

Functional enrichment analysis showed that the core F-ADGs were enriched in similar gene functions associated with the $G$ protein-coupled receptor signalling pathway, synaptic transmission and calcium ion homeostasis in the frontal cortex, temporal cortex and entorhinal cortex.

In the frontal cortex, the 18 core F-ADGs were enriched in biological processes such as $\mathrm{G}$ protein-coupled receptor signalling, the neuropeptide signalling pathway and leukocyte chemotaxis. In the temporal cortex, the 20 core F-ADGs were enriched in G protein-coupled receptor signalling and acetylcholine signalling. In the entorhinal cortex, the 32 core F-ADGs were enriched in biological processes such as $G$ protein-coupled receptor signalling, the inflammatory response, positive regulation of ion transport and enteric nervous system development.

\section{Pathway enrichment analysis of core F-ADGs}

Pathway enrichment analysis revealed that the core F-ADGs in Cluster 1 were enriched in similar pathways associated with synapse-related signalling pathways in the frontal cortex, temporal cortex and entorhinal cortex. 
In the frontal cortex, core F-ADGs were enriched in pathways such as GABAergic synapses, morphine addiction and circadian entrainment. In the temporal cortex, core F-ADGs were enriched in pathways such as GABAergic synapses, morphine addiction, and serotonergic synapses. In the entorhinal cortex, core FADGs were enriched in pathways such as GABAergic synapses, morphine addiction, glutamatergic synapses, and neuroactive ligand-receptor interactions.

\section{Correlation between core F-ADG expression and AD severity in the frontal cortex}

The correlation between the MMSE score of 9 AD patients, which ranged from 11 to 22 , were collected, and S1PR1 expression levels in the frontal cortex were analysed. We found that the expression level of FPR1 was strongly correlated with MMSE scores, GNAI1, NPY2R and NPY expression levels were significantly correlated, and S1PR1, GPR183 and GNG5 expression levels showed weak linear correlations with MMSE scores. The correlation between a lower MMSE score and more severe AD suggests that S1PR1, GNG5, FPR1 and GPR183 expression levels are positively correlated with the severity of AD, while NPY, NPY2R and GNAI1 expression levels are negatively correlated with the severity of AD.

\section{Analysis of immune cell infiltration in the cortices of $A D$ patients}

Immune cell infiltration was analysed based on gene expression data from the frontal cortices, temporal cortices and olfactory cortices of AD patients. No difference in immune cell infiltration was observed between $A D$ patients and normal controls in any of the cortical regions. This phenomenon is consistent with typical cognitive and pathological observations of $A D$ patients, suggesting that the expression changes in S1PR1 and other fingolimod targets identified in this study were mainly due to changes in cells in the CNS rather than changes in immune cell infiltration in the CNS.

\section{Discussion}

$A D$ is the most common cause of dementia in the world, and there are no effective therapies that can halt disease progression[1][2][3]. The S1PR modulator fingolimod (FTY720), which is an immunomodulator used in the treatment of multiple sclerosis, was recently reported to be effective in the treatment of $A D$, but its pharmacological mechanisms remain elusive. While fingolimod has been shown to have effects on APP metabolism and $A \beta$ protein aggregation in animal models of $A D$, the potential mechanisms underlying its effects in $A D$, such as synaptic functional changes, neuronal survival, synaptic plasticity, neuronal transmission, and axonal growth, have not yet been validated. It is also uncertain whether these potential effects of fingolimod are specific to AD or nonspecific effects in the CNS.

In this study, we found that some of pharmacological targets of fingolimod, such as S1PR1 and S1PR3, and predicted pharmacological targets, such as GNG11 and GABBR2, were differentially expressed in multiple cortical regions in patients with $A D$ compared with normal controls and were enriched in GABA synapse-related signalling pathways. This finding has two implications. First, S1PR1 is associated with abnormal function of GABA synapses in AD. Second, S1PR1 may be a drug target for the regulation of abnormal synaptic function in AD. Furthermore, since the expression level of S1PR1 was found to be 
positively correlated with the severity of AD in this study, drugs targeting S1PR1 could influence the development of $A D$.

Fingolimod, as an analogue of S1P, induces downstream G protein-coupled receptor pathways by inducing the invagination of S1PRs on the cell surface and regulates downstream signalling factors and biological processes[4][5]. The main isoforms of S1PR that are expressed in the brain are S1PR1, S1PR2, S1PR3, and S1PR5, among which S1PR1 binds most strongly with fingolimod[6][7]. Based on the fact that the expression of S1PR1, its downstream factors Fox01 and AKT1[8][9], and some G protein-coupled receptor-related cytokines was higher in multiple cortical regions in $A D$ patients than in normal controls, fingolimod may have a stronger effect on the brain tissues of AD patients than on those of normal controls and may affect the pathological processes of AD by targeting S1PR1.

In this study, it was found that S1PR1 was in the functional core cluster and enriched in pathways associated with GABA synapses, suggesting that the 'influence of fingolimod on S1PR1 in AD brain tissue can influence pathological changes in GABA synaptic function. Notably, oral administration of fingolimod has been reported to alter synaptic function in the brain tissues of experimental autoimmune encephalomyelitis (EAE) mice[10][11], but it was not clear whether this change is due to alterations in the immune response or a direct effect on brain tissue. Based on the general elevation of S1PR1 expression in the cortices of $A D$ patients compared to normal controls observed in this study and the fact that inflammatory cell infiltration in $A D$ is significantly lower than that in multiple sclerosis and $E A E$, the role of fingolimod in AD may mainly be derived from its direct effects on the CNS and may be related to the regulation of GABAergic synaptic function. Furthermore, increased expression of S1PR3, which was also enriched in GABAergic synaptic functions, was detected in the entorhinal cortices of AD patients compared to those of normal controls, suggesting that S1PR3 is also involved in the processes of AD and may be a therapeutic target of fingolimod. Since the entorhinal cortex is often the most seriously affected cortex in $A D[12][13]$, we believe that as $A D$ progresses, various S1PRs may be involved in the development of $A D$; that is, the effect of fingolimod on $A D$ may involve a variety of S1PR pathways.

CCKBR and CHRM1 were detected in the temporal cortex and entorhinal cortex and enriched in gene functions such as calcium signalling regulation and cholinergic synapses. Calcium signalling is necessary for many biological functions and is also necessary for synaptic release, which is involved in various synaptic processes such as synaptic homeostasis and synaptic plasticity[14][15]. In this study, genes associated with calcium signalling regulation that were abnormally expressed in the brains of patients with $A D$ were found to be targets of fingolimod, suggesting that fingolimod could play a therapeutic role in $A D$ by regulating calcium signalling. This study also revealed that cholinergic synaptic dysfunction-related genes associated with $A D$ could be targeted by fingolimod. Based on this study, various marker proteins of the cholinergic system are altered in the cortices of AD patients; specifically, the activities of choline acetyltransferase (ChAT) and acetylcholine esterase (AChE), the release of acetylcholine (Ach) and receptor expression, which are correlated with the severity of dementia, are reduced[16][17]. A lack of choline in neuronal progenitors affects the expression of thousands of genes, most of which are involved in cell proliferation, differentiation, and methyl metabolism[18][19]. Currently, 
cholinergic synaptic function is the main drug target for $A D$ treatment; for example, the drug donepezil hydrochloride is a cholinergic drug that is used to treat $A D[20][21]$. This study revealed that fingolimod may affect the cholinergic pathway in $A D$, indicating another mechanism by which fingolimod may be used to treat $A D$.

Notably, some of the core F-ADGs are associated with the targeting of HTR2C and HTR2A rather than S1PRs by fingolimod, suggesting that fingolimod may have targets other than S1PRs in the brain. Moreover, fingolimod but not the active form of the drug, fingolimod phosphate, affects these other targets. It is generally believed that fingolimod does not directly excite S1PRs but is phosphorylated by SPHK2 to target S1PRs[22]. Since unlike fingolimod phosphate, fingolimod can cross the BBB, it is generally believed that fingolimod is phosphorylated to form fingolimod phosphate in the CNS, suggesting that there are at least two forms of fingolimod present in the CNS[23][24]. This study suggests that unphosphorylated fingolimod may play a pharmacological role in the CNS through receptors other than S1PRs, such as HTR2C and HTR2A, and influence the occurrence and development of AD.

Other target genes of fingolimod in addition to the core F-ADGs were found to be differentially expressed in the brain tissues of $A D$ patients compared to those of normal controls, suggesting that fingolimod may affect pathological processes of $A D$ other than those associated with the core F-ADGs. Fingolimod has been shown to increase BDNF levels in primary cortical neurons in mice, suggesting that fingolimod may alleviate the effects of a lack of BDNF in $A D[25]$. In this study, the expression of BDNF in the temporal cortices of $A D$ patients was significantly lower than that in the temporal cortex of normal controls, suggesting that increased expression of BDNF by fingolimod may play a role in AD. Fingolimod may also be phosphorylated by SPHK2 and inhibit class I histone deacetylases (HDACs), thereby promoting histone acetylation and inducing gene expression changes[26][27][28][29][30]. In this study, it was found that HDAC1 and HDAC4 expression was higher in the temporal entorhinal cortices of AD patients than those of normal controls, suggesting that the inhibitory effect of fingolimod on HDACs may alleviate AD. In addition, fingolimod has been shown to reduce TNF-induced expression of B-cell activating factor (BAFF) and chemokine interferon-y inducible protein 10 (CXCL10), which have immunoregulatory effects, in astrocytes[31]. This study revealed that the expression of CXCL10 was higher in the frontal cortices of $A D$ than those of normal controls, suggesting that the abnormal increase in CXCL10 expression in $A D$ is involved in the development and progression of the disease and that fingolimod could play a role in immune regulation in $\mathrm{AD}$-affected brains.

Finally, to explore whether the identified core genes originate in the CNS or from immune cell infiltration of brain tissue, we analysed immune infiltration in different brain tissues from $A D$ patients and normal controls. No significant difference was detected between the AD patients and normal controls, suggesting that there was no obvious immune cell infiltration in the brain tissues examined in this study; this finding is consistent with the current understanding of AD pathology. While some recent studies have found that infiltration of neutrophils and other cells can occur in the frontal cortices of AD patients[32][33], we did not observe this phenomenon in the samples examined in this study. Therefore, we believe that the core 
factors (such as S1PR1) and signalling pathways identified in this study are derived from cells in the CNS, such as neurons and glial cells, rather than infiltrating peripheral immune cells.

\section{Limitations}

This study has a number of limitations. First, this study is a bioinformatics analysis, and the predicted

fingolimod drug targets are only potential drug targets. Whether these potential targets can be targeted by fingolimod in the real environment, and the size of the impact on the pathogenesis of AD after being targeted still needs further animal and clinical trials to verify. Meanwhile, since the samples included in this study come from multiple databases, some clinical data and MMSE scores may have different standards, which may cause bias and affect the results of the study. Therefore, in the future, more largescale and strictly limited clinical studies of AD patients receiving fingolimod treatment are necessary.

\section{Conclusion}

The pharmacological macromolecular targets of fingolimod and fingolimod phosphate, such as S1PR1, were abnormally expressed in multiple cortical regions in $A D$ patients and to be correlated with $A D$ severity. The pharmacological mechanism underlying the effect of fingolimod on AD in the CNS may be attributed to its ability to regulate GABA synapses, calcium signalling, and cholinergic synapses, other than regulating immune cell infiltration.

\section{Abbreviations}

$A D$ : Alzheimer's disease

S1PR: sphingosine-1-phosphate receptor

FGs: fingolimod-associated genes

ADGs: AD-associated genes

F-ADGs: fingolimod-associated ADGs

MCODE: Minimal Common Oncology Data Elements

PPI: protein-protein interaction

GABA: Gamma-Aminobutyric Acid

CNS: central nervous system

CSF: Cerebrospinal fluid

FDA: Food and Drug Administration 
RRMS: relapsing-remitting multiple sclerosis

BBB: blood-brain barrier

APP: $\beta$-amyloid precursor protein

$A \beta$ : $\beta$-amyloid

FC: Frontal Cortex

TC: Temporal Cortex

$E C$ : Entorhinal Cortex

GO: Gene Ontology

KEGG: Kyoto Encyclopaedia of Genes and Genomes

MMSE: Mini-Mental State Exam

EAE: experimental autoimmune encephalomyelitis

ChAT: choline acetyltransferase

AChE: acetylcholine esterase

Ach: acetylcholine

HDACs: histone deacetylases

CXCL10: chemokine interferon-y inducible protein 10

BAFF: B-cell activating factor

\section{Declarations}

\section{Availability of data and materials}

The datasets used and/or analysed during the current study are available from the corresponding author on reasonable request.

\section{Acknowledgements}

We thank the participants and their families for their generous support of this study.

\section{Funding}


Not applicable

\section{Contributions}

The study was conceived and designed by Pengqi Yin, Yang Xue and Guozhong Li. Data collection was carried out by Pengqi Yin. Processing and analysis of the imaging data was performed by Pengqi Yin and Yang Xue. Pengqi Yin led the statistical analysis. All authors contributed to interpretation of the data and preparation of the manuscript. The authors read and approved the final manuscript.

\section{Corresponding author}

Correspondence to Di Zhong and Guozhong Li.

Ethics approval and consent to participate

Not applicable

Consent for publication

Not applicable

Competing interests

No potential conflict of interest was reported by the authors.

\section{Tables}

Due to technical limitations, table 1 is only available as a download in the Supplemental Files section.

\section{References}

Reference list not available with this version.

\section{Figures}




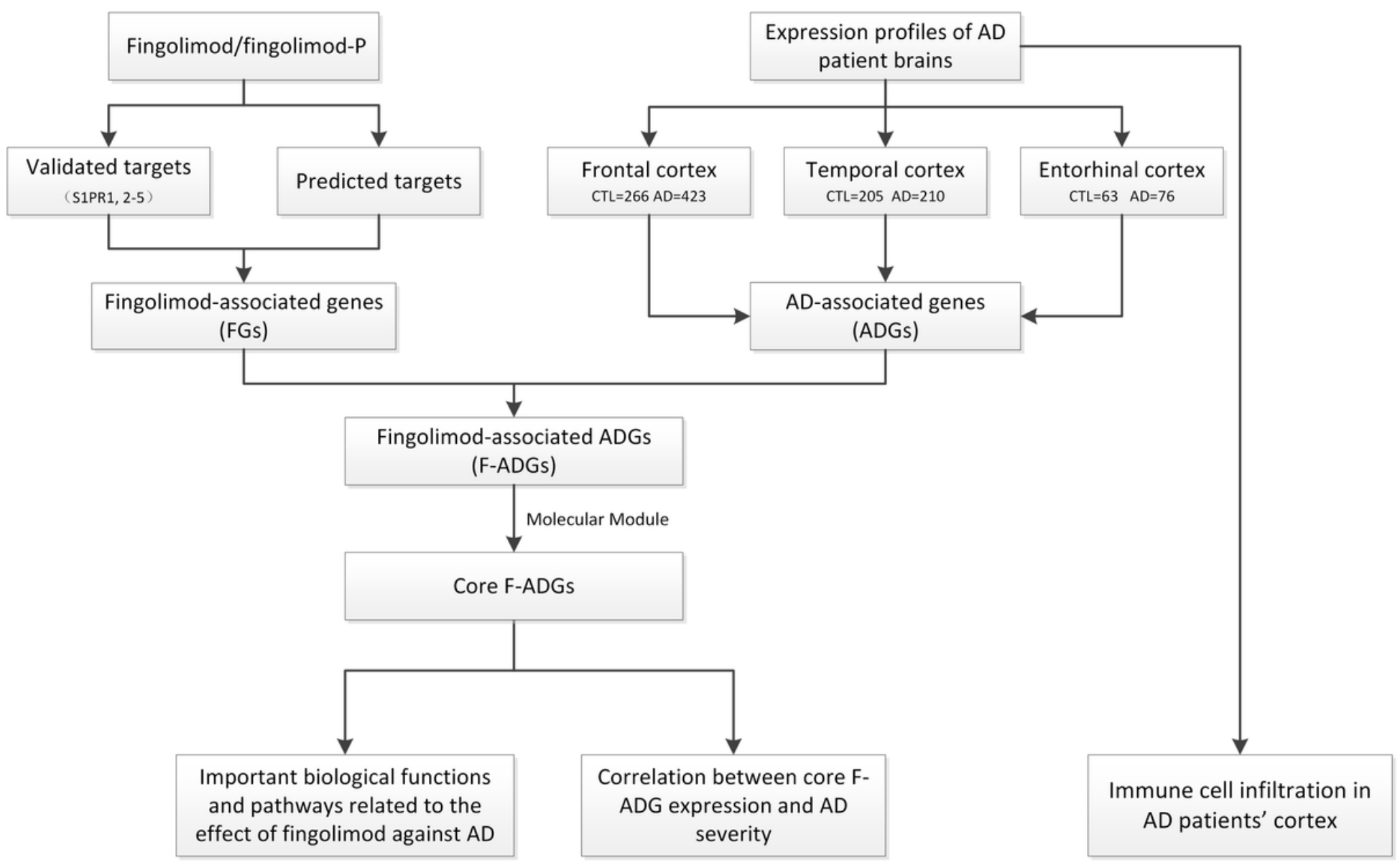

\section{Figure 1}

Flowchart of the analysis of the effect of fingolimod against AD. AD: Alzheimer's disease; CTL: control; Fingolimod-P: fingolimod phosphate; FGs: fingolimod-associated genes; ADGs: AD-associated genes; FADGs: fingolimod-associated AD-associated genes.
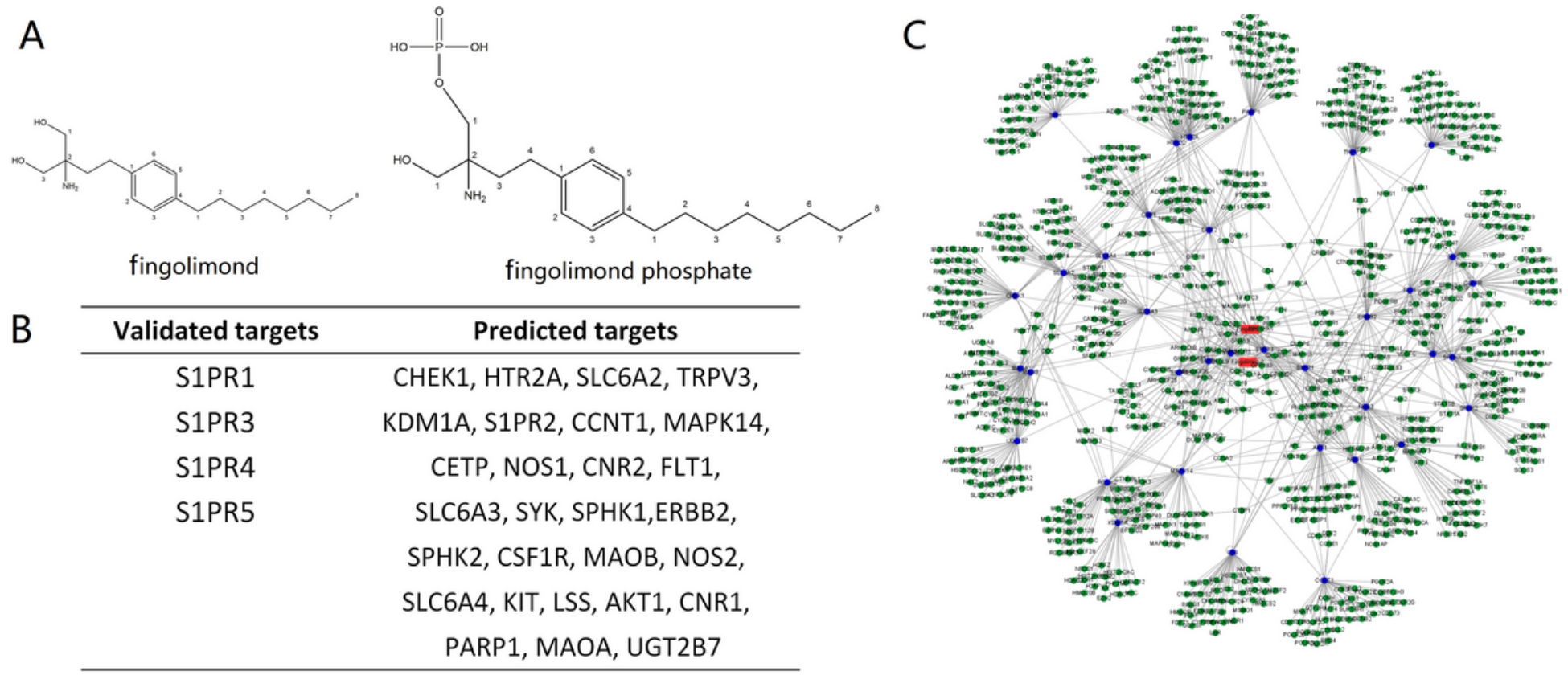
Pharmacological fingolimod targets and FGs (a) The chemical structure of fingolimod and fingolimod phosphate. (b) The pharmacological targets of fingolimod and fingolimod phosphate. (c) FGs, including the pharmacological targets of fingolimod and fingolimod phosphate (blue dots) and the proteins that potentially interact with these pharmacological targets (green dots). The red square represents fingolimod and its metabolite fingolimod phosphate.

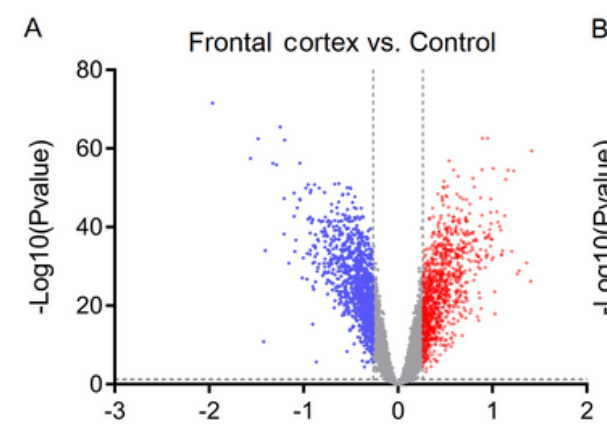

Log2(FoldChange)

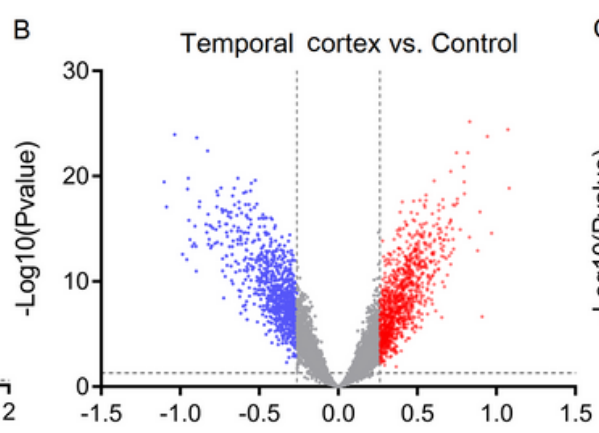

Log2(FoldChange)

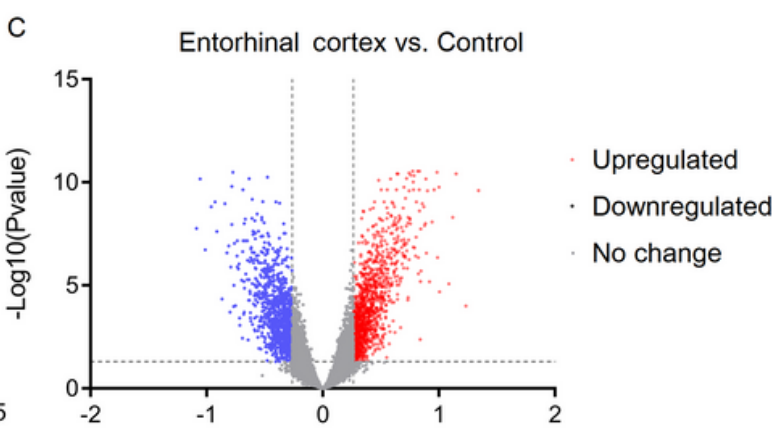

Log2(FoldChange)

\section{Figure 3}

Differentially expressed genes in the brain cortices of $A D$ patients compared to those of normal controls. Volcano map showing the differentially expressed genes in the cortices of AD patients compared to those of normal controls. Significantly upregulated genes are shown in red, significantly downregulated genes are shown in blue and unchanged genes are shown in gray. (a) Frontal cortex. (b) Temporal cortex. (c) Entorhinal cortex.

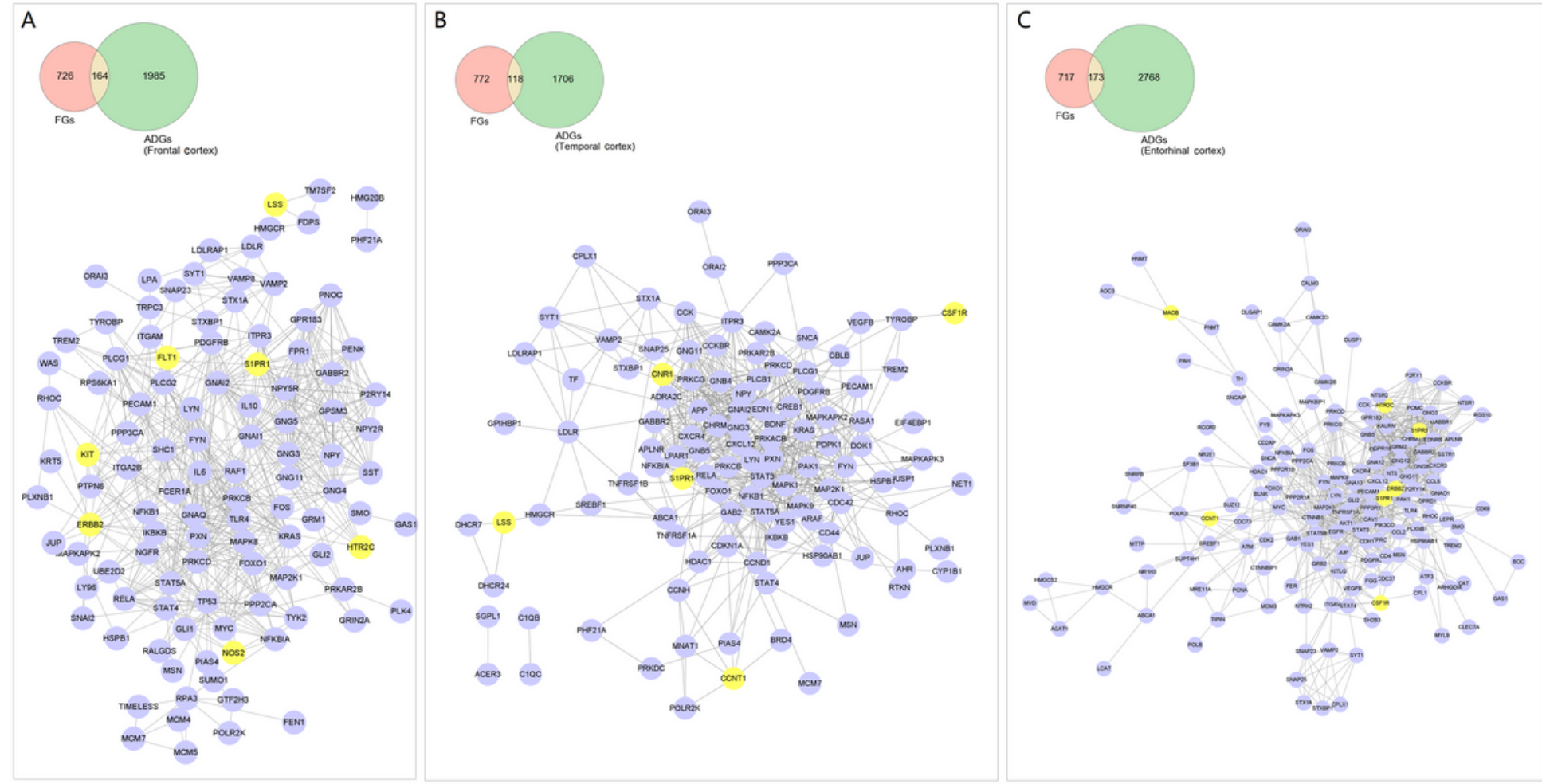

\section{Figure 4}


F-ADGs in the different cortical regions of AD patients. (a) Frontal cortex. (b) Temporal cortex. (c) Entorhinal cortex. Venn diagrams showing the number of F-ADGs. Each node in the PPI network represents an F-ADG. The nodes in yellow represent confirmed targets of fingolimod.

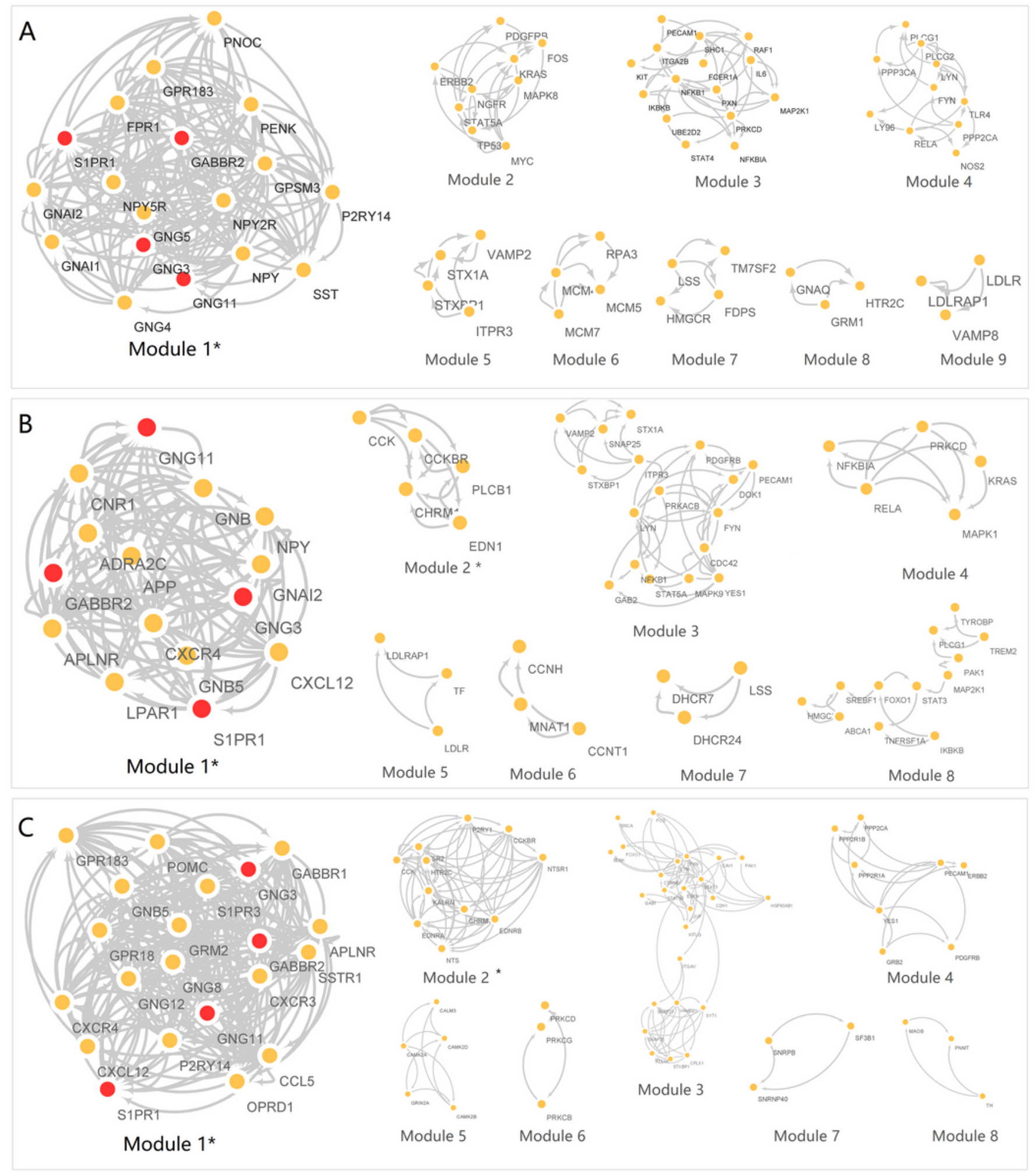

\section{Figure 5}

Molecular modules generated by Cytoscape MCODE analysis of PPI networks in different cortical regions of AD patients. (a) Frontal cortex. (b) Temporal cortex. (c) Entorhinal cortex. Each node represents an F- 
ADG. Core modules were screened based on the criterion twice the average degree of MCODE score and are indicated by an asterisk ${ }^{*}$ ). Red nodes represent genes (core F-ADGs) in core modules in all three cortical regions.

A
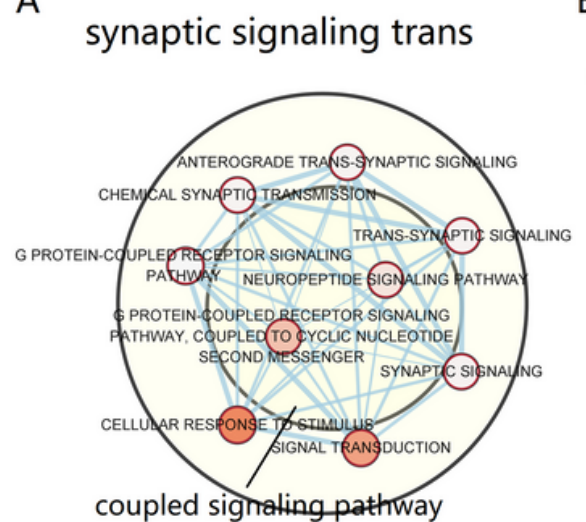

B homeostasis positive regulation

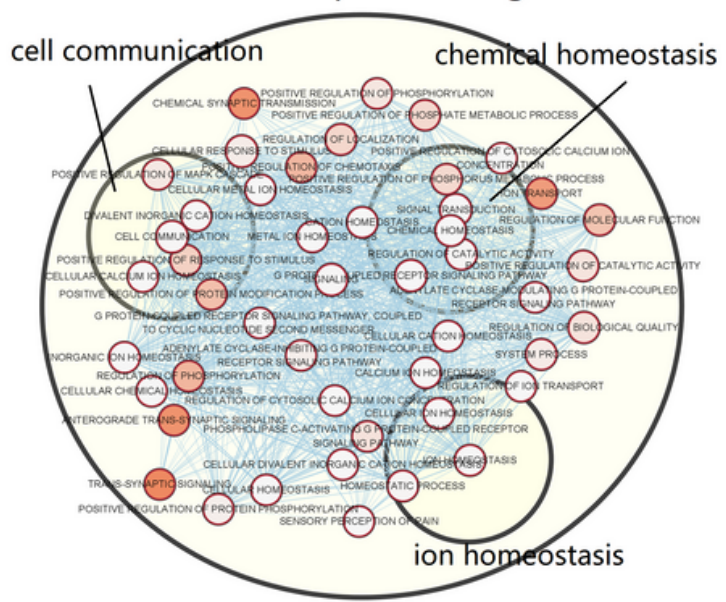

C ion homeostasis regulation

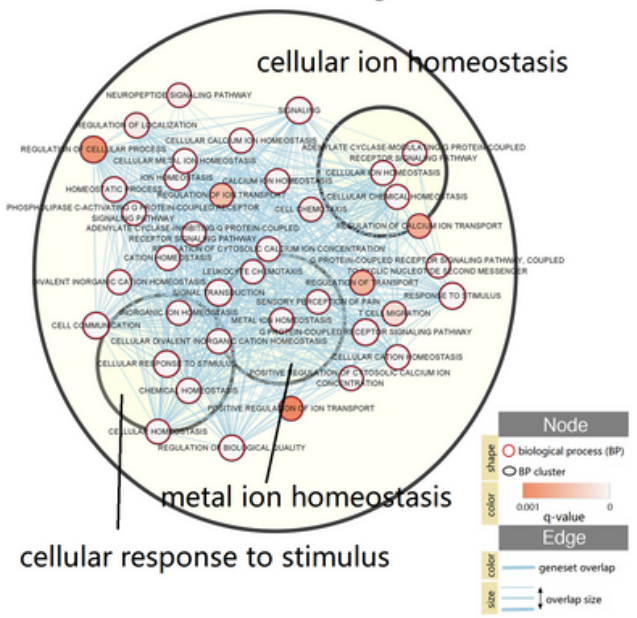

Figure 6

Biological process modules generated by the Cytoscape Enrichment Map of g:profiler results for different stages of AD. (a) Frontal cortex. (b) Temporal cortex. (c) Entorhinal cortex. An enrichment map was created based on the parameters $q<0.1$ and Jaccard overlap combined coefficient $>0.35$. The nodes represent enriched biological processes, which are grouped and annotated based on their similarity according to connected component modules. 

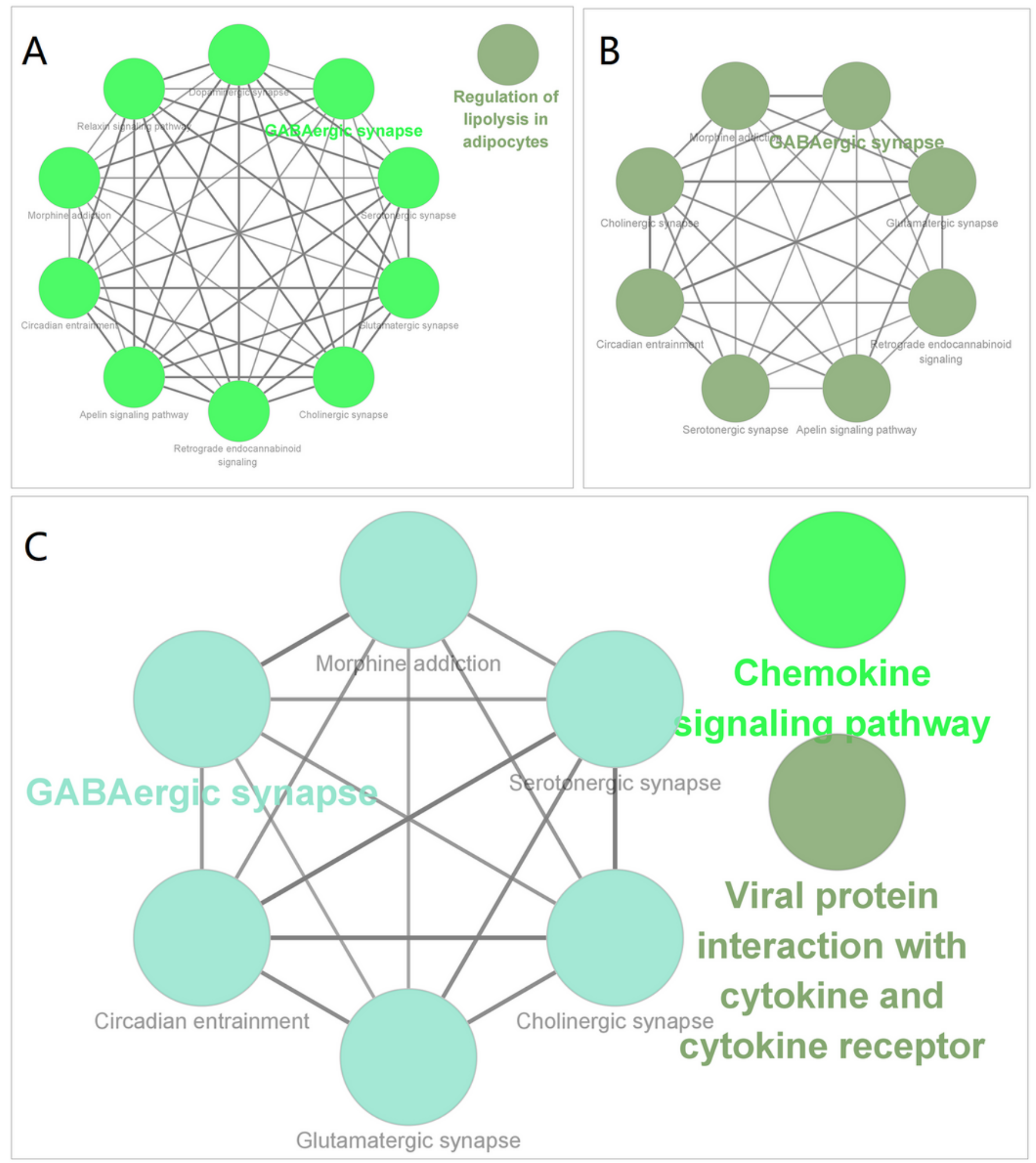

\section{Figure 7}

KEGG pathways enriched among genes differentially expressed in the frontal cortex (a), temporal cortex (b) and entorhinal cortex (c) in AD patients. Each node represents a pathway. The node size indicates the significance of the pathway; pathways with smaller false discovery rates (FDRs) have larger nodes at different AD stages. The different node colours represent different functional groups. The most 
significant pathway in each group is highlighted. The edge between nodes reflects shared or common genes; a wider edge indicates a larger overlap.
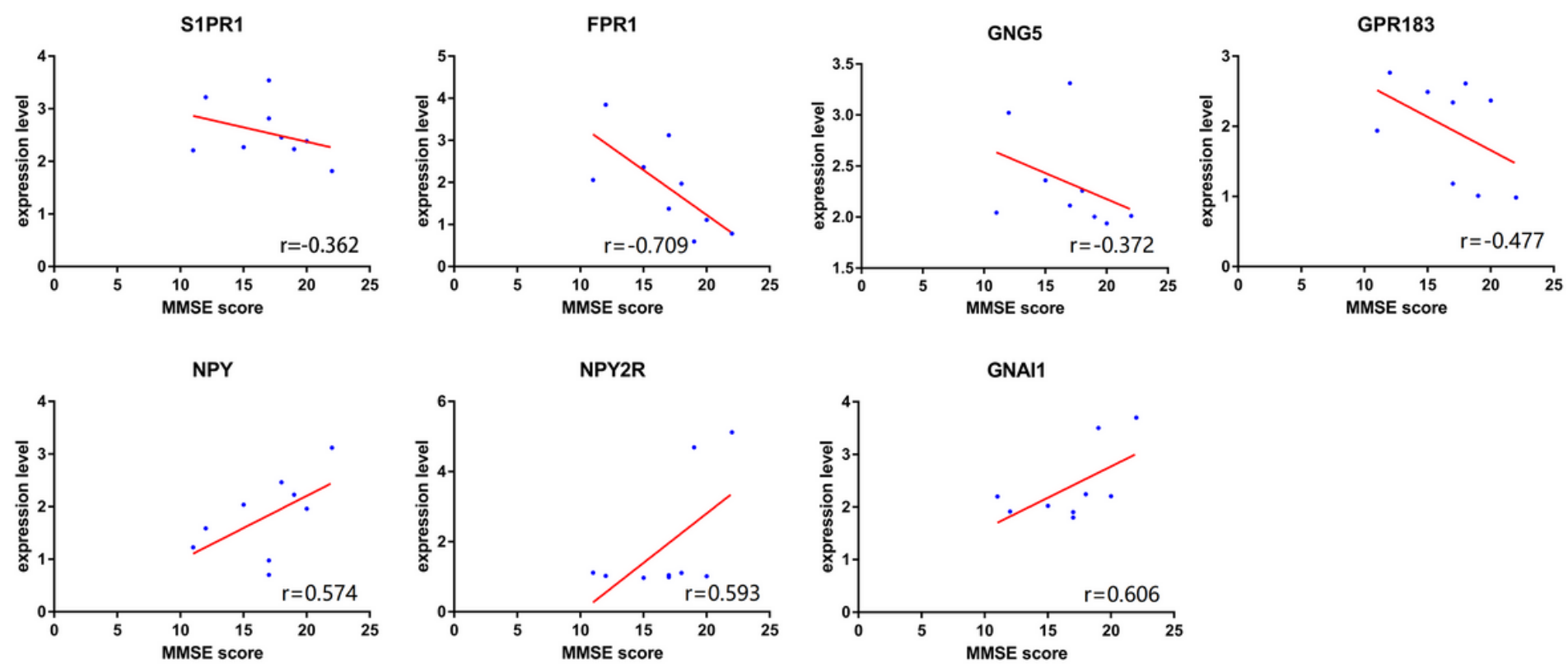

\section{Figure 8}

Correlation analysis of the expression levels of core F-ADGs and MMSE scores as a measure of AD clinical severity. MMSE scores are plotted on the x-axis. Gene expression values are plotted on the $y$-axis. The red dots indicate the expression levels of the core genes in each sample. 
A

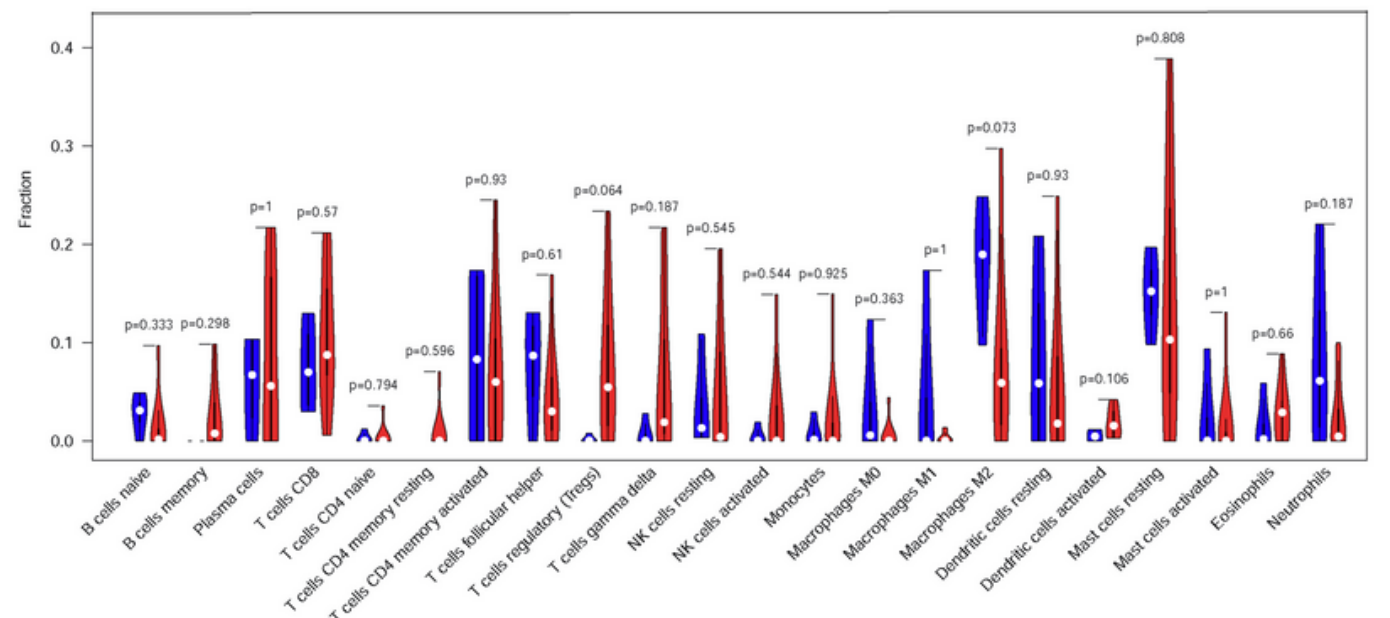

B

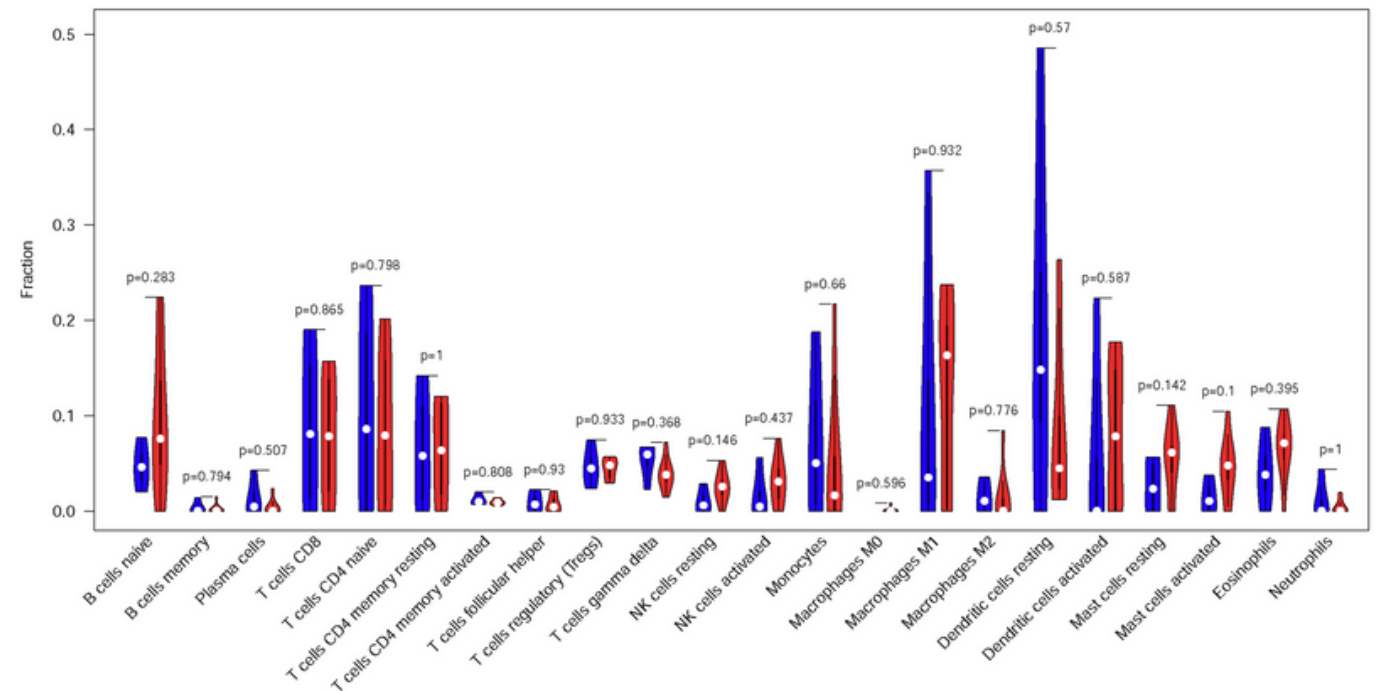

C

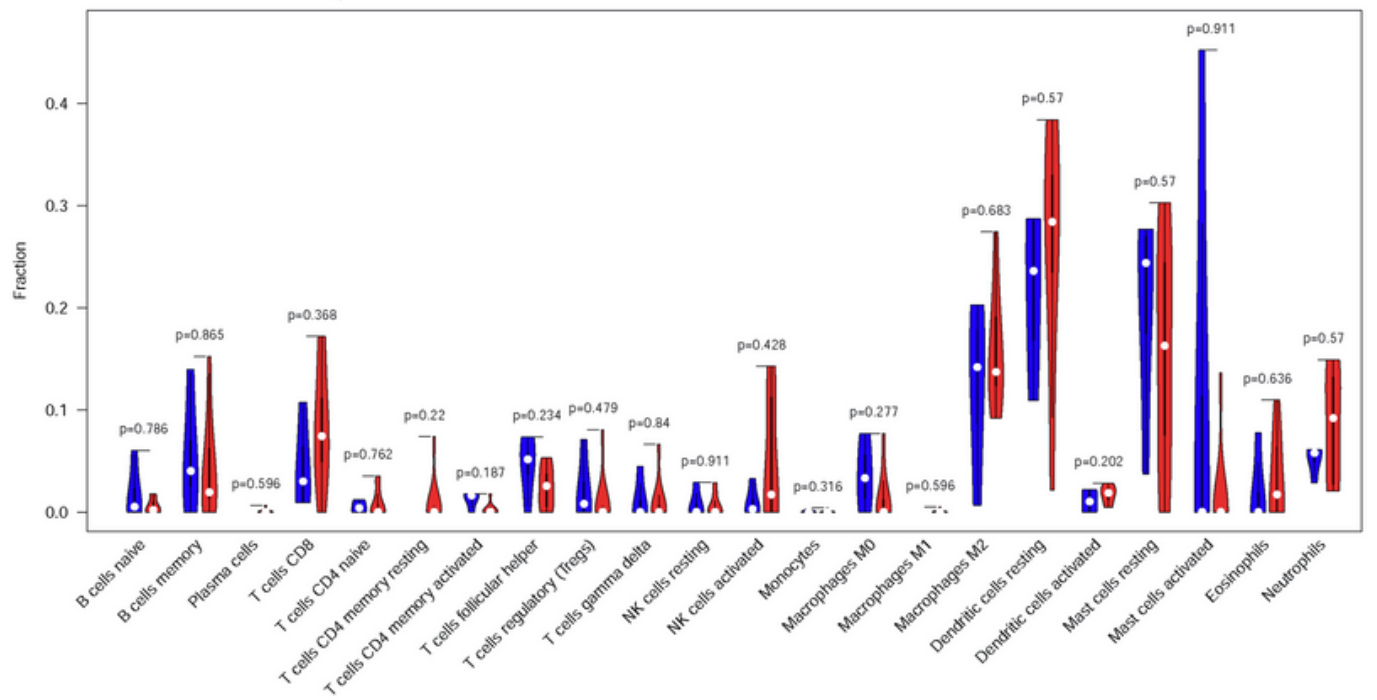

Figure 9

Immune cell infiltration in the cortices of AD patients. (a) Frontal cortex. (b) Temporal cortex. (c) Entorhinal cortex. Immune cell type is plotted on the x-axis. The fraction of immune cell content is plotted on the $y$-axis. The red bars in the violin plots indicate the fraction of immune cell infiltration in $A D$ patients, and the blue bars in the violin plots indicate the fraction of immune cell infiltration in normal controls. 


\section{Supplementary Files}

This is a list of supplementary files associated with this preprint. Click to download.

- Table1.xlsx 